\title{
Practical Aspects of X-ray Imaging Polarimetry of Supernova Remnants and Other Extended Sources
}

\author{
Jacco Vink ${ }^{1,2, *}$ (D) and Ping Zhou ${ }^{3,4}$ \\ 1 GRAPPA \& Anton Pannekoek Institute for Astronomy, University of Amsterdam, Science Park 904, \\ 1098 XH Amsterdam, The Netherlands \\ 2 SRON, Netherlands Institute for Space Research, Sorbonnelaan 2, 3584 CA Utrecht, The Netherlands \\ 3 Anton Pannekoek Institute for Astronomy, University of Amsterdam, Science Park 904, \\ 1098 XH Amsterdam, The Netherlands; p.zhou@uva.nl \\ 4 School of Astronomy and Space Science, Nanjing University, Nanjing 210023, China \\ * Correspondence: j.vink@uva.nl; Tel.: +31-20-525-7068
}

Received: 23 February 2018; Accepted: 29 March 2018; Published: 10 April 2018

\begin{abstract}
The new generation of X-ray polarisation detectors, the gas pixel detectors, which will be employed by the future space missions IXPE and eXTP, allows for spatially resolved X-ray polarisation studies. This will be of particular interest for X-ray synchrotron emission from extended sources like young supernova remnants and pulsar wind nebulae. Here we report on employing a polarisation statistic that can be used to makes maps in the Stokes $I, Q$, and $U$ parameters, a method that we expand by correcting for the energy-dependent instrumental modulation factor, using optimal weighting of the signal. In order to explore the types of Stokes maps that can be obtained, we present a Monte Carlo simulation program called xpolim, with which different polarisation weighting schemes are explored. We illustrate its use with simulations of polarisation maps of young supernova remnants, after having described the general science case for polarisation studies of supernova remnants, and its connection to magnetic-field turbulence. We use xpolim simulations to show that in general deep, $\sim 2$ Ms observations are needed to recover polarisation signals, in particular for Cas A, for which in the polarisation fraction may be as low as $5 \%$.
\end{abstract}

Keywords: X-rays; polarimetry; supernova remnants; cosmic ray; particle acceleration; magnetic-field turbulence; monte-carlo simulations; individual objects: Cas A, SN 1572, SN 1006

\section{Introduction}

The polarisation of electromagnetic radiation is an important diagnostic tool to determine the nature of the radiation mechanisms as well as obtaining information on the geometry and radiative transfer properties of radiation in and near the source of radiation. $X$-ray polarisation is in that sense no different, but has the added value that $\mathrm{X}$-ray emission comes from high-energy sources with extreme conditions. For young supernova remnants (SNRs) and pulsar wind nebulae (PWNe), X-ray polarisation holds the promise to determine the geometry and turbulent properties of the magnetic fields, which determines the polarisation fraction and orientation of the magnetic fields, in case that the radiation is caused by $\mathrm{X}$-ray synchrotron emission. These are also properties that can be assessed through radio polarisation structures, but $\mathrm{X}$-ray synchrotron comes from the highest energy electrons ( $>10 \mathrm{TeV}$, e.g., [1]), which are generally short lived ( $\lesssim 100 \mathrm{yr}$ ) and, therefore, sample plasmas that are still near the sites where the electrons have been accelerated. Since acceleration of charged particles depends on the strength and the turbulence of the magnetic field, X-ray polarisation holds a great promise in determining the conditions under which the electrons have been accelerated. 
X-ray polarisation studies have been done in the past, but without the ability of imaging polarisation structures. They typically made use of the linear polarisation dependence of Thomson/Compton scattering, or were based on scattering by a Bragg crystal. For extended sources the absence of imaging capabilities is far from ideal as inside the sources there are likely to be regions with different polarisation properties, diminishing or completely wiping out the overall polarisation fraction that can be measured. Nevertheless, for the Crab nebula a net X-ray polarisation signal was measured with sounding rocket experiments [2] and Bragg crystal polarimeters on board the OSO-8 satellite [3,4]. For the Crab nebula the overall X-ray polarisation fraction is $\Pi=19 \%$ with a position angle of $154^{\circ}$ [4], in agreement with optical and radio observations [5,6]. However, more detailed maps in the radio [7] and optical [8] show regions with much higher polarisation fraction, and even evolution of polarisation as a function of time for the inner regions [8]. It is this level of imaging detail that has been lacking in X-ray polarisation observations so far, but this is going to change in the near future.

The ability to image X-ray polarisation has been brought about with the development of polarisation sensitive gas pixel detectors (GPDs) $[9,10]$, which makes use of the photoelectric effect in a thin layer of gas, and the fact that the photoelectron ejection angle $\alpha$ is modulated as $\cos ^{2}$ with respect to the polarisation vector of the X-ray emission. The photoelectron creates a cascade of secondary free electrons, whose positions are recorded. From the location and shape of the electron cloud the original ejection angle has to be reconstructed.

This new type of X-ray polarisation detectors has led to new proposals for satellite based X-ray polarisation missions: the X-ray Imaging Polarimetry Explorer (XIPE) [11] for ESA, the Imaging X-ray Polarisation Explorer (IXPE) [12] for NASA, and the enhanced X-ray Timing and Polarimetry Mission (eXTP) [13] for the Chinese space science program. XIPE was considered for the ESA M4 science program, but it was recently announced that XIPE did not make the final selection ${ }^{1}$. A decision that was to be expected, as NASA did select the very similar IXPE mission for its small mission program. It uses for example the same GPD detectors. IXPE is expected to be launched in 2021/22. Also eXTP uses this type of detector, but the eXTP mission combines X-ray polarisation instruments together with X-ray timing detectors. eXTP faces this year (2018) a final review, and if successful could be launched around $2025^{2}$.

The purpose of this article is to explore the imaging capabilities of X-ray imaging polarimeters based on GPDs, with a special focus on young SNRs for which the expected polarisation signals are weaker than for PWNe. We start with explaining the use of the photon-by-photon statistics method (e.g., [14]), which has obvious advantages over the more traditional fitting of modulation curves (e.g., Figure 10 in [15]) when it come to polarisation imaging, as no extraction regions need to predefined, and one can just make maps in Stokes $\mathrm{Q}$ and $\mathrm{U}$, and its derivates (polarisation fraction $\Pi$, and polarisation angle). We then describe a Monte Carlo code, which helps to define optimum observation strategies for young SNRs and $\mathrm{PWNe}$, such as observation times and energy band optimisation. It also allows us to assess what the effects are of the point spread function on the polarisation signals. We then explain what X-ray polarisation measurements can teach us about particle acceleration in young SNRs, and what kind of signal to expect.

Some earlier results were already reported in [16], but here we provide new results and a new scheme for correcting for the instrumental modulation factor.

\section{Measuring X-ray Polarisation with Gas-Pixel-Detectors on a Photon by Photon Statistics Base}

Gas-pixel-detectors (GPDs) to be used by the IXPE [12] and eXTP [13] satellite experiments do not measure directly the polarisation of the electric vector, but rather the direction of the photon-electron,

See http:/ / sci.esa.int/cosmic-vision/59796-esa-s-next-science-mission-to-focus-on-nature-of-exoplanets.

See http:/ / www.sciencemag.org/news/2018/03/china-unveils-plans-x-ray-satellite-probe-most-violent-corners-universe. 
which is ejected from an atom in the gas-filled detector, if ionised by an X-ray photon. The angle $\alpha$ of the direction of the photon-electron is $\cos ^{2} \alpha$ modulated with respect to the associated electric-field vector of the photon.

In X-ray polarisation studies, traditionally the polarisation is measured by binning the measured angles, $\alpha_{i}$, and then fitting the function

$$
M(\alpha)=\left[1-\Pi+2 \Pi \cos ^{2}\left(\alpha-\alpha_{0}\right)\right] \sin (2 \alpha),
$$

to the binned data, using a $\chi^{2}$ minimisation method.

It is easy to apply (1) for non-imaging devices, as one simply bins the measured events per angle $\alpha$ and fit the histogram. However, with GPDs one would like to make optimum use of the imaging capabilities. Using (1) would then require predefining an image region of interest, and bin the $\alpha$ for all events in that region. Or, if the statistics is sufficient, do this for every pixel. But doing the binning and minimisation for each pixel separately is computationally expensive.

However, there is an easier method, which does not require binning the data for ejection angle $\alpha$.

The method is laid out in Kislat et al. [14] and we explain it here, but using slightly different notations. As estimators for the Stokes parameters $I, Q, U$ we use:

$$
\begin{gathered}
\hat{I} \equiv \sum_{i=1}^{i=N} 1=N \\
\hat{Q} \equiv 2 \sum_{i=1}^{i=N} \cos \left(2 \alpha_{i}\right), \\
\hat{U} \equiv 2 \sum_{i=1}^{i=N} \sin \left(2 \alpha_{i}\right) .
\end{gathered}
$$

The angle $\alpha_{i}$ will depend on the true polarisation direction $\alpha_{0}$ as $\cos ^{2}\left(\alpha-\alpha_{0}\right)$. It is a matter of taste whether one takes the average (divide $\hat{Q}$ and $\hat{U}$ by $N$ ) or the plain sum as shown here. In the absence of any polarisation signal the expectation values are $E \hat{Q}=E \hat{U}=0$.

The equation is almost similar to the definition of the $Q$ and $U$ Stokes parameters, except that there is a factor 2, which accounts for the fact that not the electric-vector, but a $\cos ^{2}$ modulated statistical realisation of it is measured. This factor was omitted in the definition of $Q$ and $U$ in [14], but there it accounted for when calculating the true polarisation fraction.

The measured polarisation fraction is given by

$$
\Pi=\frac{\sqrt{\hat{Q}^{2}+\hat{U}^{2}}}{\hat{I}} .
$$

We use here the same symbol $\Pi$ as for the modulation amplitude (1), because they are essentially the same. For example the expectation value of $\hat{Q}$ for a signal that varies as $M(\alpha)(1)$ is

$$
\begin{aligned}
E(\hat{Q}) & =\frac{2}{2 \pi} \int_{0}^{2 \pi}\left[(1-\Pi)+2 \Pi \cos ^{2}\left(\alpha-\alpha_{0}\right)\right] \cos (2 \alpha) d \alpha=0+\frac{1}{\pi} \int_{0}^{2 \pi} \Pi\left[1+\cos \left(2 \alpha-2 \alpha_{0}\right)\right] \cos (2 \alpha) d \alpha \\
& =0+\Pi \frac{1}{\pi} \int_{0}^{2 \pi}\left[\cos ^{2}(2 \alpha) \cos \left(2 \alpha_{0}\right)+\cos (2 \alpha) \sin (2 \alpha) \sin \left(2 \alpha_{0}\right)\right] d \alpha \\
& =\Pi \frac{1}{\pi}\left\{\cos \left(2 \alpha_{0}\right) \int_{0}^{2 \pi} \cos ^{2}(2 \alpha) d \alpha+\frac{1}{2} \sin \left(2 \alpha_{0}\right) \int_{0}^{2 \pi} \sin (4 \alpha) d \alpha\right\}=\Pi \cos \left(2 \alpha_{0}\right) .
\end{aligned}
$$

And similarly, $E(\hat{U})=\Pi \sin \left(2 \alpha_{0}\right)$. Equation (3) provides an estimate for the true polarisation factor, if there are no instrumental depolarisation effects. How to correct for the instrumental effects will be discussed below. 
The variance for $\hat{Q}$ and $\hat{U}$ can be directly estimated assuming Poisson statistics:

$$
\begin{aligned}
& \operatorname{Var}(\hat{Q}) \equiv 4 \sum_{i=1}^{i=N} \cos ^{2}\left(2 \alpha_{i}\right), \\
& \operatorname{Var}(\hat{U}) \equiv 4 \sum_{i=1}^{i=N} \sin ^{2}\left(2 \alpha_{i}\right) .
\end{aligned}
$$

The expectation values (see also [14]) in the absence of a polarisation signal are $E \operatorname{Var}(\hat{Q})=$ $E \operatorname{Var}(\hat{U})=2 N$. Note that here we estimate the variances from the measured event data itself, whereas in [14] one makes use of the expectation values for estimating the variances, assuming no polarisation is present.

The advantage of using (5) for X-ray imaging polarimetry are obvious: for each imaging pixel $\hat{Q}$ and $\hat{U}$ and their variances can be easily calculated. If the signals are noisy one can just rebin the $\hat{I}$, $\hat{Q}$ and $\hat{U}$ maps, because rebinning is summing pixels together, which just is equivalent to calculating $\hat{Q}$ and $\hat{U}$ using more events.

However, the formalism does not take into account that the measured angles $\alpha_{i}$ contain errors. These angles reduce the polarisation signal. If a hypothetical source would be $100 \%$ polarised $\Pi=1$ the measured polarisation signal will be $\Pi=\mu<1$, with $\mu$ the so-called modulation factor, which is energy-dependent factor (Figure 1).

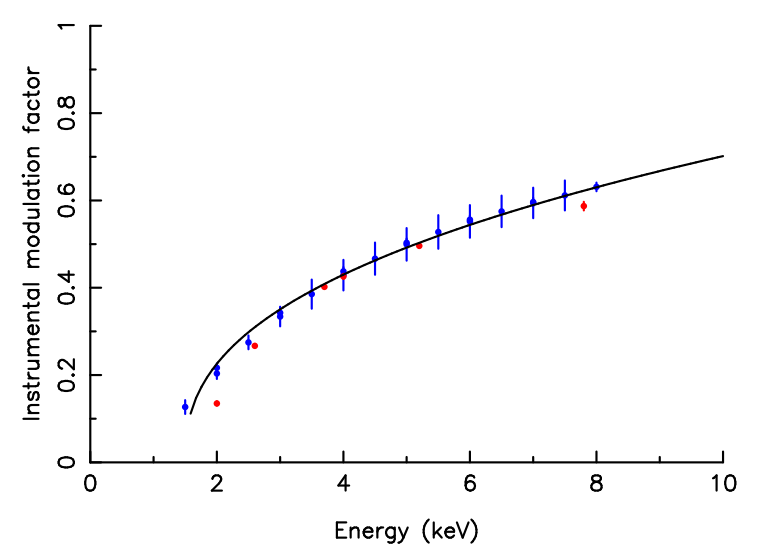

Figure 1. The modulation factor, $\mu$, as a function of energy, i.e., the measured polarisation fractions for a $100 \%$ polarised source, not correcting for instrumental effects. The red points are measurements taken from Muleri et al. [15]. The blue points are based on the Monte Carlo code described in this text, and uses a Gaussian error $\Delta \alpha \propto \sqrt{E}$ for the photo-electron ejection angle. The curved line is an analytical approximation for the modulation factor, used for the corrections on a event-by-event base.

If $\hat{Q}$ and $\hat{U}$ would be determined in a narrow energy band an estimate of the polarisation fraction of the source itself is

$$
\hat{\Pi}=\frac{1}{\mu} \frac{\sqrt{\hat{Q}^{2}+\hat{U}^{2}}}{\hat{I}} .
$$

In practice one may want to use all available events rather than selecting a narrow energy band, which suggests that we correct each individual event with its energy-specific modulation factor $\mu_{i}$ :

$$
\begin{aligned}
\hat{Q}_{\text {corrected }} & \equiv 2 \sum_{i=1}^{i=N} \frac{1}{\mu_{i}} \cos \left(2 \alpha_{i}\right), \\
\hat{U}_{\text {corrected }} & \equiv 2 \sum_{i=1}^{i=N} \frac{1}{\mu_{i}} \sin \left(2 \alpha_{i}\right),
\end{aligned}
$$




$$
\begin{aligned}
& \operatorname{Var}\left(\hat{Q}_{\text {corrected }}\right) \equiv 4 \sum_{i=1}^{i=N}\left(\frac{1}{\mu_{i}}\right)^{2} \cos ^{2}\left(2 \alpha_{i}\right), \\
& \operatorname{Var}\left(\hat{U}_{\text {corrected }}\right) \equiv 4 \sum_{i=1}^{i=N}\left(\frac{1}{\mu_{i}}\right)^{2} \sin ^{2}\left(2 \alpha_{i}\right) .
\end{aligned}
$$

This would indeed provide an estimate of the source polarisation itself, but it has one disadvantage: It is essentially a weighted sum, with $\frac{1}{\mu_{i}}>1$ as a weight. If the modulation factor is small, i.e., the instrument/energy specific polarisation performance is poor, we give that event more weight.

Instead what we would like is to make a weighted sum that still provides a proper estimate of the true polarisation factor, but that optimises the polarisation signal to noise ratio. From a statistical point of view the optimum weighting scheme is to use inverse variance weighting which uses weights $w_{i}=1 / \sigma_{i}^{2}[17,18]$. Inspecting Equation (7) shows that if we were to use narrow energy bins the variance of the signal scales with $1 / \mu_{i}^{2}$, which implies that we take as weights $w_{i}=\mu_{i}^{2}$. With these weights applied the estimator that optimises the signal to noise becomes

$$
\begin{aligned}
\hat{I}_{\text {optimal }} & \equiv N \frac{\sum_{i=1}^{i=N} \mu_{i},}{\sum_{i=1}^{i=N} \mu_{i}^{2},} \\
\hat{Q}_{\text {optimal }} & \equiv 2 N \frac{\sum_{i=1}^{i=N} \mu_{i} \cos \left(2 \alpha_{i}\right)}{\sum_{i=1}^{i=N} \mu_{i}^{2}}, \\
\hat{U}_{\text {optimal }} & \equiv 2 N \frac{\sum_{i=1}^{i=N} \mu_{i} \sin \left(2 \alpha_{i}\right)}{\sum_{i=1}^{i=N} \mu_{i}^{2}}, \\
\operatorname{Var}\left(\hat{Q}_{\text {optimal }}\right) & \equiv 4 N^{2} \frac{\sum_{i=1}^{i=N} \mu_{i}^{2} \cos ^{2}\left(2 \alpha_{i}\right)}{\left(\sum_{i=1}^{i=N} \mu_{i}^{2}\right)^{2}}, \\
\operatorname{Var}\left(\hat{U}_{\text {optimal }}\right) & \equiv 4 N^{2} \frac{\sum_{i=1}^{i=N} \mu_{i}^{2} \sin ^{2}\left(2 \alpha_{i}\right)}{\left(\sum_{i=1}^{i=N} \mu_{i}^{2}\right)^{2}} .
\end{aligned}
$$

which if all $\mu_{i}$ would be identical would give exactly the same result as Equation (7). The multiplication factors $N$ (and $N^{2}$ for the variances) can be omitted, but serve here to make that the definition of the Stokes I parameter, $\hat{I}$, remains identical to Equation (5). For convenience we drop the subscript "optimal", and write $\Delta \hat{Q} \equiv \sqrt{\operatorname{Var}(\hat{Q})}$ and $\Delta \hat{U} \equiv \sqrt{\operatorname{Var}(\hat{U})}$.

The estimators $\hat{Q}, \hat{U}, \operatorname{Var}(\hat{Q}), \operatorname{Var}(\hat{U})$ contain all the information necessary. In practice one would like to quantify the polarisation signal in terms of the polarisation fraction

$$
\hat{\Pi} \equiv \frac{\sqrt{\hat{Q}^{2}+\hat{U}^{2}}}{\hat{I}}
$$

and position angle of the polarisation vector

$$
\phi=\frac{1}{2} \arctan \left(\frac{\hat{U}}{\hat{Q}}\right) .
$$

One has to be careful here when reporting these two quantities and their errors, as both quantities are based on a combination of $\hat{Q}, \hat{U}$ and are, therefore, correlated. In the literature one often encounter the term polarisation intensity, which is simply the intensity in polarised light only $\hat{I}_{\text {pol }}=\sqrt{\hat{Q}+\hat{U}}=\Pi \hat{I}$.

To find the signal-to-noise ratio of the polarisation measurement we calculate first the error in $\sqrt{\hat{Q}^{2}+\hat{U}^{2}}$ : 


$$
\Delta\left(\sqrt{\hat{Q}^{2}+\hat{U}^{2}}\right)=\left[\left(\frac{\partial \sqrt{\hat{Q}^{2}+\hat{U}^{2}}}{\partial \hat{Q}} \Delta \hat{Q}\right)^{2}+\left(\frac{\partial \sqrt{\hat{Q}^{2}+\hat{U}^{2}}}{\partial \hat{U}} \Delta \hat{U}\right)^{2}\right]^{1 / 2}=\frac{\sqrt{\hat{Q}^{2}(\Delta \hat{Q})^{2}+\hat{U}^{2}(\Delta \hat{U})^{2}}}{\sqrt{\hat{Q}^{2}+\hat{U}^{2}}} .
$$

The signal-to-noise ratio for the polarised intensity is therefore

$$
S / N=\frac{\sqrt{\hat{Q}^{2}+\hat{U}^{2}}}{\Delta\left(\sqrt{\hat{Q}^{2}+\hat{U}^{2}}\right)}=\frac{\hat{Q}^{2}+\hat{U}^{2}}{\sqrt{\hat{Q}^{2}(\Delta \hat{Q})^{2}+\hat{U}^{2}(\Delta \hat{U})^{2}}} .
$$

Note that the method advocated in [14] is to use for the variance the expectation value for the variances in absence of a polarisation signal. Since in the absence of a signal the expectation value for the variance is maximised the method in [14] is a little bit more conservative.

We consider estimates based on Equation (9) as the preferred statistic for measuring polarisation. Note that one may use the weights/modulation factors based on measured calibration results as depicted in Figure 1, but that in the future estimates for the weights $1 / \mu_{i}^{2}$ may be based on estimated errors for each individual event; for example on how easily $\alpha_{i}$ could be determined from the electron cloud morphology.

Finally we point out that the method outlined here for making maps in $Q$ and $U$ can also be used for timing and spectral bins in order to make polarisation lightcurves and polarisation spectra.

\section{A Monte Carlo Code for X-ray Gas Pixel Detectors}

The GPDs allow for the imaging of X-ray polarisation structures of the extended sources like PWNe and SNRs. However, a problem we encounter for SNRs is that the polarisation fraction may be low (see Section 4). Moreover, as we will discuss below, the X-ray synchrotron emitting regions are in some cases, like Cas A, confined to small regions near the forward (and reverse) shocks, whereas from the rest of the SNR shell, non-polarised, thermal emission is emitted. The ratio of X-ray synchrotron emission over non-thermal emission varies from SNR to SNR; for Cas A, Kepler's SNR, and Tycho's SNR most of the X-ray emission is thermal in nature, and the X-ray synchrotron emission is best studied in the 4-6 keV band, in which there is almost no line emission. For studies with GPDs that means that the analysis has to be confined to this narrow band, which limits the statistics. For SN 1006 (but also RXJ1713etc and Vela Jr) the synchrotron emitting regions are broader, but also the X-ray synchrotron emission dominates the overall emission, certainly above $2 \mathrm{keV}$. Although these SNRs have a lower X-ray surface brightness, the larger energy bandwidth partially compensates for this.

To study the effects of the energy band used, the low polarisation fractions to be expected based on the radio observations, and to study the effects of the point spread function (PSF) on mixing photons from thermally emitting regions with non-thermally emitting regions, it is best to resort to Monte Carlo simulations of the expected signals. Moreover, a Monte Carlo code allows us to test the statistical methods explained in Section 2. We set out to make a Monte-Carlo code to test imaging with the XIPE mission, but since IXPE and EXTP will carry similar detectors and telescopes, the results will be relevant to all three missions. The set-up of the Monte-Carlo code, named $x^{2}$ lim $^{3}$ is illustrated in Figure 2.

The code uses as an input a high resolution $\mathrm{X}$-ray image of the object, for example a Chandra image of Cas A in the 4-6 keV band. Based on a spectral model (for now the code only deals with power-law spectral models) and the instruments effective area (mirror plus detector) the number of detect photons and their energy distribution is predicted for a given observation time. The photon arrival directions are randomly drawn from the input image, and based on a toy model of the polarisation direction

3 Currently xpolim is not publicly available, but an executable for MacOS will be made available on request by the J. Vink. Depending on the demand and availability of funding for further development of xpolim, we will work toward a documented and portable version of the source code. 
and polarisation fraction a photon-electron angle $\alpha$ is assigned to the event. The values $\alpha$ takes into account the $\cos ^{2}$ modulation based on the physics of the photo-electric effect, and in addition a random Gaussian error is added, which represents that the initial direction of the photo-electron cannot be accurately determined from the electron cloud. Since the number of electrons scales with the energy as $N_{\text {pe }} \propto E$, and the error on reconstruction is probably partially Poissonian in nature, we assumed that $\Delta \alpha \propto \sqrt{N_{\mathrm{pe}}} \propto \sqrt{E}$. After some experimenting with the Monte-Carlo simulations we found that

$$
\Delta \alpha=5^{\circ}+35^{\circ} \sqrt{\frac{E}{4 \mathrm{keV}^{\prime}}}
$$

gives a good fit to the measured modulation factor above $2 \mathrm{keV}$ reported in [15] (see Figure 1). We used this $\Delta \alpha$, but also its associated modulation factor, represented by the analytical function

$$
\mu=29.8(E-1.5 \mathrm{keV})^{0.4} \%
$$

for $E>1.5 \mathrm{keV}$, otherwise $\mu=0$. This functional form, which is used to estimate the actual polarisation signal using (9), is shown as a solid line in Figure 1.

INPUT

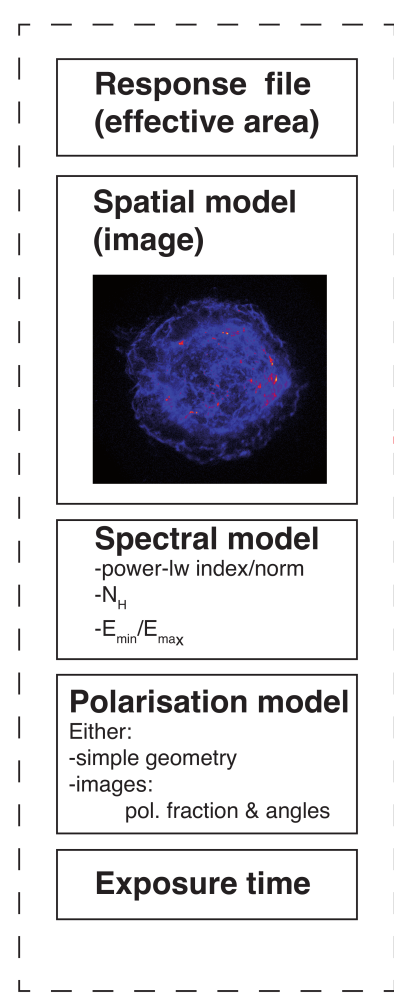

MONTE CARLO
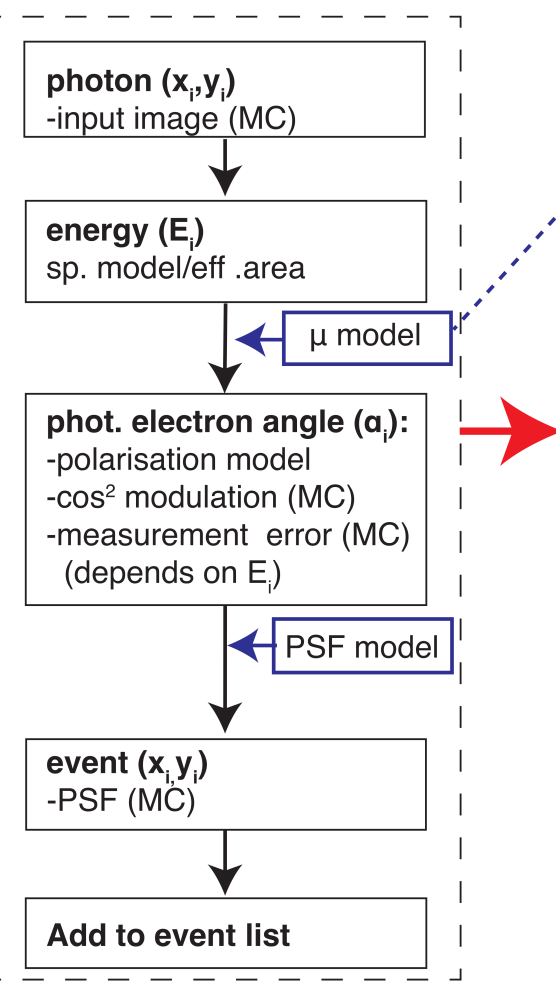

EVENTS PROCESSING

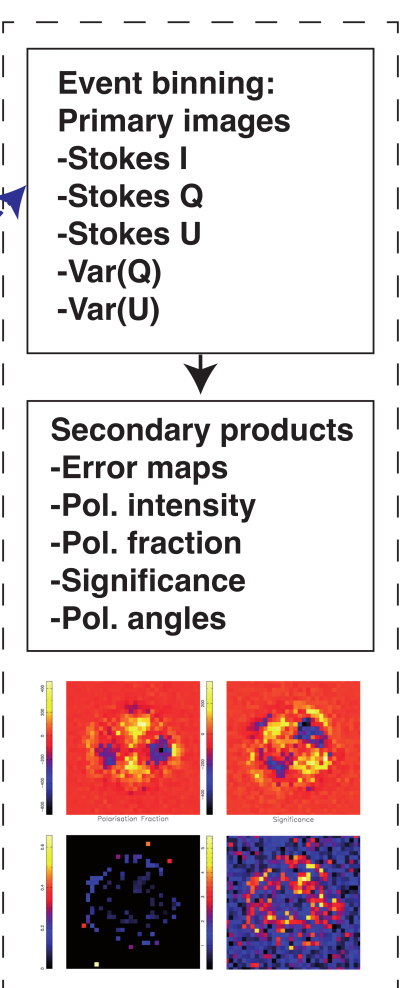

Figure 2. Schematic view of the xpolim code.

After assigning a photoelectron angle to each event, the event itself is subject to a random realisation of the PSF, for which we used the analytical description reported in [19], which is based on calibration measurements of the GPD in combination with X-ray optics.

The output of the xpolim program is an event list, which can then be processed according to the statistical method described in Section 2. In fact, all three methods (no weighting, correction for $\mu$ and a weighted correction) are implemented, and can be tested. The output itself consists of binned images of the Stokes $I, Q, U$ and their variances, as well as their derived products: the polarisation fraction, its error, the polarisation detection significance (15) and reconstructed polarisation angles. 
Currently spectral redistribution of the events based on a response file is not yet implemented, and the input spectral models are limited to power-law distributions modified by a simple interstellar absorption model [20].

\section{The Expected X-ray Polarisation Signals from Shell-Type Supernova Remnants}

The realisation that the radio emission from SNRs was caused by synchrotron emission from relativistic electrons, provided the first observational evidence for a link between cosmic-ray acceleration and supernovae [21]. Since long the mechanism to accelerate the electrons and protons to relativistic energies is generally accepted to be diffusive shock acceleration (DSA) (for a review [22]). Radio synchrotron emission is caused by accelerate electrons with a few $\mathrm{GeV}$ of energies, whereas the cosmic rays of Galactic origin extend all the way up to the cosmic-ray "knee" at $3 \times 10^{15} \mathrm{eV}$. To accelerate to these high energies, magnetic fields have to be considerably stronger than the average Galactic magnetic field strength of $\sim 5 \mu \mathrm{G}$, and the magnetic field needs to be highly turbulent. In fact according to DSA theory the magnetic-field turbulence is created by the cosmic rays themselves, as they diffuse ahead of the shock front. A process that has drawn substantial theoretical attention, but for which the observational data is still scarce (e.g., $[23,24])$.

The discovery of X-ray synchrotron emission from shell-type SNRs, first for SN 1006 [25], but later for virtually all SNRs younger than 1000-2000 yr (for a review [1]), showed that electrons can be accelerated to very high energies of $10^{13}-10^{14} \mathrm{eV}$, which requires in fact very high levels of turbulence and high shock velocities. The synchrotron cut-off photon energy for synchrotron radiation should occur around [26]

$$
h v_{\max } \approx 3\left\langle\left(\frac{\delta B}{B}\right)^{2}\right\rangle\left(\frac{V_{\mathrm{sh}}}{5000 \mathrm{~km} \mathrm{~s}^{-1}}\right)^{2} \mathrm{keV} .
$$

This equation is derived by setting the acceleration rate equal to the energy-dependent synchrotron loss rate of the electrons. The electron synchrotron loss time for $10-100 \mathrm{TeV}$ is very short compared to the ages of SNRs:

$$
\tau_{\text {syn }} \approx 12.5\left(\frac{B}{100 \mu \mathrm{G}}\right)^{-2}\left(\frac{E_{\mathrm{e}}}{100 \mathrm{TeV}}\right)^{-1} \mathrm{yr} .
$$

This short synchrotron loss time scale explains why X-ray synchrotron emission is only found close to the shock fronts that accelerate the particles. In fact, the thickness of the X-ray synchrotron rims can be used to estimate the magnetic field strengths [27], which appear to be 20-500 $\mu \mathrm{G}$ (for an overview [28]).

The fact that young SNRs emit X-ray synchrotron implies that the magnetic fields are not very structured $(\delta B / B \sim 1)$. Indeed, radio polarisation studies of SNRs show that young SNRs have much lower polarisation fractions than old SNRs, see Figure 3 adopted from [29]. Moreover, the magnetic field orientation in young SNRs tend to be radially oriented, whereas for old SNRs the fields are tangential [30]. The tangential fields of old SNRs can be easily understood: the shock wave compresses only the magnetic field component running perpendicular to the shock normal. The radially field orientation in young SNRs is less well understood; it may have something to do with magnetic-field amplification in the cosmic-ray precursors of SNRs [31], or caused by a selection effect on where relativistic electrons pile up downstream of the shock [32]. X-ray polarisation studies may shed some new light on this issue, as it will allow for polarisation measurement close to the shock, and establish whether the radial structure is already present there, or is caused by processes further away from the shock. 


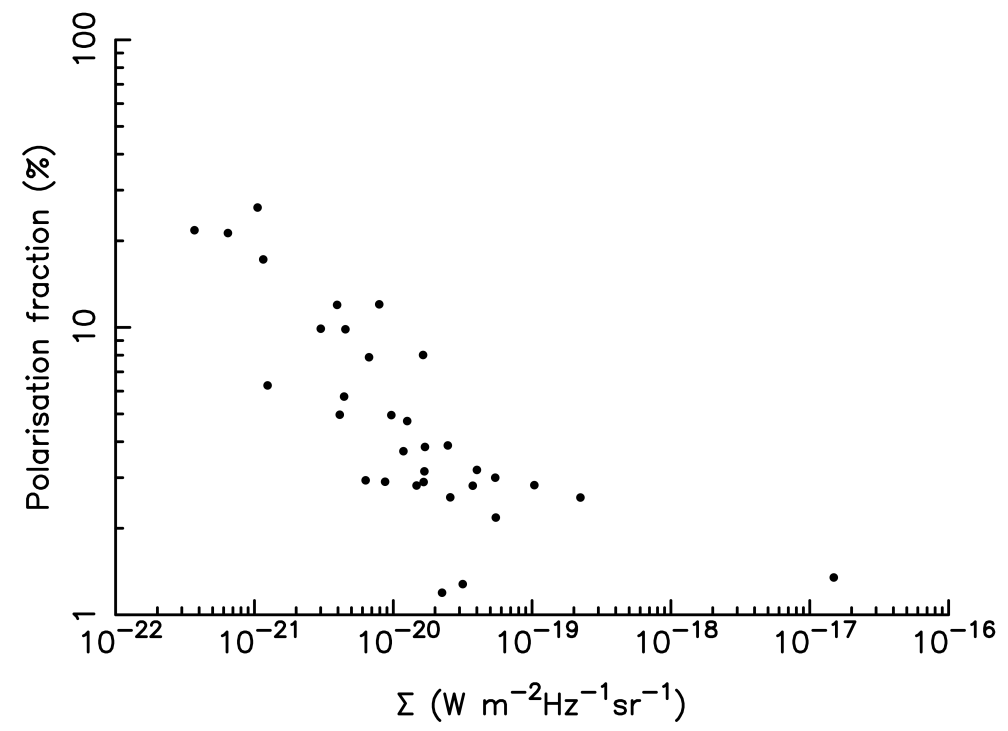

Figure 3. The radio polarisation fraction as a function of the radio surface brightness, taken from [29] and based largely on the sample in [33]. Surface brightness is here a proxy for size and/or age; low surface brightness remnants tend to be larger and older. So this plot shows that young SNRs have low polarisation fractions (few $\%$ to $\sim 20 \%$ ).

The low polarisation fraction of young SNRs in the radio, potentially makes the detection of $\mathrm{X}$-ray polarisation challenging. On the other hand, since the $\mathrm{X}$-ray synchrotron rims are confined to regions close to shocks fronts, $\mathrm{X}$-ray polarisation studies offer the opportunity to study magnetic field orientations and turbulence close to sites of active acceleration. Related to this is that the $\mathrm{X}$-ray synchrotron emitting regions occupy less volume than the radio synchrotron emitting regions. So depolarisation due to different magnetic field orientations along the line of sight is less likely in the X-rays than in the radio. So the $\mathrm{X}$-ray polarisation fraction may be higher in $\mathrm{X}$-ray than in radio. Finally, the maximum polarisation fraction depends on the spectral index of the synchrotron emission. The maximum polarisation fraction depends on the spectral photon number index, $\Gamma$, as (c.f. [34])

$$
\Pi_{\max }=\frac{\Gamma}{\Gamma+\frac{2}{3}} .
$$

So a steeper index will result in higher polarisation fractions. Since the X-ray synchrotron emission is associated with photon energies near spectral cut-off, the spectral index is usually steeper than in the radio (note that $\Gamma=\alpha+1$, with $\alpha$ the radio spectral energy index). In the radio young SNRs have typically $\Gamma \approx 1.6$, corresponding to $\Pi_{\max } \approx 70.5 \%$, whereas in X-rays $\Gamma \approx 3$, corresponding to $\Pi_{\max } \approx 82 \%$. In addition, the $\mathrm{X}$-ray synchrotron emission may be selecting out more the high magnetic field peaks, where the higher magnetic fields push the cut-off energies into the X-ray band. This effectively reduces the volume of the $\mathrm{X}$-ray synchrotron emitting plasma even further, enhancing the polarisation fraction. All effects discussed so far suggest that $X$-ray synchrotron polarisation fraction may be higher than radio polarisation. However, the proximity of X-ray synchrotron radiation regions to the shock front, may preferentially select regions with high magnetic-field turbulence. So this could result in lower polarisation fractions in X-rays as compared to the radio, for which the emission comes on average from regions further away from the shock front. The magnitude of the magnetic-field fluctuations and their size distributions has been described quantitatively in [35], who show that X-ray polarisation studies are an important means to study the magnetic field fluctuations that are so important for a proper understanding of DSA in young SNRs. 
In the next section we show what the detection possibilities are for polarisation in young SNRs, using the typical polarisation fractions based on the radio polarisation studies (Table 1).

Table 1. Radio polarisation measurements of young SNRs.

\begin{tabular}{ccccccc}
\hline Object & Typical $\Pi$ & Peak $\boldsymbol{\Pi}$ & Orientation $\boldsymbol{B}$ & $\lambda$ & Remarks & Ref. \\
\hline RCW 86 & $8 \%$ & $15 \%$ & radial & $22 \mathrm{~cm}$ & some regions $\Pi<3 \%$ & {$[36]$} \\
SN 1006 & $17 \%$ & $60 \%$ & mostly radial & $20 \mathrm{~cm}$ & peak not in X-ray rims & {$[37]$} \\
SN 1572 & $7 \%$ & $25 \%$ & radial/fine struct. $\left(4^{\prime \prime}\right)$ & $6 \mathrm{~cm}$ & peak at limbs & {$[38]$} \\
SN 1604 & $6 \%$ & $12 \%(?)$ & radial/fine struct. $\left(20^{\prime \prime}\right)$ & $6 \mathrm{~cm}$ & & {$[39]$} \\
Cas A & $5 \%$ & $\sim 20 \%$ & radial & $6 \mathrm{~cm}$ & outer plateau $\Pi \approx 9 \%$ & {$[40,41]$} \\
G1.9 0.3 & $6 \%$ & $17 \pm 3 \%$ & radial & $6 \mathrm{~cm}$ & Faraday rotation? & {$[42]$} \\
\hline
\end{tabular}

\section{Simulations of Supernova Remnants}

The purpose of the set of simulations we will describe in this section is twofold: (1) it should inform us on what what type of results to expect from future X-ray polarisation missions and what exposure times can realistically expected to result in polarisation detection; and (2) help us test and experiment with data analysis techniques.

For the input polarisation models we simply specified a location-dependent polarisation fraction and direction. For a discussion of the polarisation effects of underlying magnetic field turbulence on the X-ray polarisation signal we refer to [35]. The two methods can be combined as shown in Figures 2 and 3 of the XIPE yellowbook ${ }^{4}$.

We present here simulations of three SNRs: Cas A, Tycho (SN1572), and SN1006. For the simulations we made educated guesses about what the polarisation fraction may be in different locations of the remnants, based on radio polarisation measurements (Table 1). But we emphasise again that the X-ray synchrotron polarisation may be different, as regions close to shocks are sampled, and the line-of-sights are shorter. The simulations were done assuming the effective area and PSF of XIPE, which has characteristics similar to IXPE and eXTP. The input spectral parameters are listed in Table 2.

Table 2. Spectral power-law parameters used for the simulations.

\begin{tabular}{ccccc}
\hline Object & $\Gamma$ & Norm & $\begin{array}{c}\text { Band Simulated } \\
\left(\mathbf{p h ~ s} \mathbf{~ s}^{\mathbf{1}} \mathbf{k e V}^{-\mathbf{1}} @ \mathbf{1} \mathbf{~ k e V )}\right.\end{array}$ & $\begin{array}{c}\text { Reference } \\
\mathbf{( k e V )}\end{array}$ \\
\hline Cas A & 3.32 & 2.37 & $4-6$ & {$[27]$} \\
SN 1572 & 3.2 & 0.77 & $4-6$ & Based on Chandra data \\
SN 1006 & 2.73 & 0.0405 & $2-8$ & {$[43]$} \\
\hline
\end{tabular}

\subsection{Cas $A$}

Cas $\mathrm{A}$ is a $\sim 340$ year old SNR, has a diameter of $5.5^{\prime}$, and is one of the brightest radio sources in the sky. Its X-ray synchrotron filaments are very narrow $\sim 1-2^{\prime \prime}$ [27] and, unique among SNRs, seems to be associated both with the forward and the reverse shock $[44,45]$.

The radio polarisation fraction of Cas $\mathrm{A}$ is unusually low, $5 \%$, and the magnetic fields are radially oriented throughout the shell, but perhaps with some deviations near the forward shock [46]. A concern for X-ray polarisation measurements of Cas A is that the bright shell and interior X-ray filaments smear out photons to the projected locations of the narrow filaments at the forward shock. In order to test whether this would wipe-out the polarisation signal at the forward shock, we set-up the polarisation

4 https://www.cosmos.esa.int/documents/1365222/1365271/SCI-2017-4+XIPE.pdf/1943687e-4d31-af62-ab791b0e1445ef14. 
in a way that maximises the depolarisation effects of the PSF: we assume that the magnetic field at the outer filaments is perpendicular (polarisation vectors radial), whereas for the interior region the polarisation vectors are perpendicular. On the other hand we assume, perhaps somewhat optimistically, that the outer filaments are characterised by a polarisation fraction of $15 \%$, whereas for the interior filaments it is assumed to be $5 \%$ (close to the radio polarisation fractions).

The input image was the Chandra 2004 image [47] in the 4-6 keV continuum band (the blue part in Figure 4, left), and also the simulated output is for that energy band. Given the narrow range in photon energy, the effects of the different weighting schemes is small. The simulated exposure times were $2 \mathrm{Ms}$, necessary to obtain sufficient signal to noise per pixel in the narrow energy band.
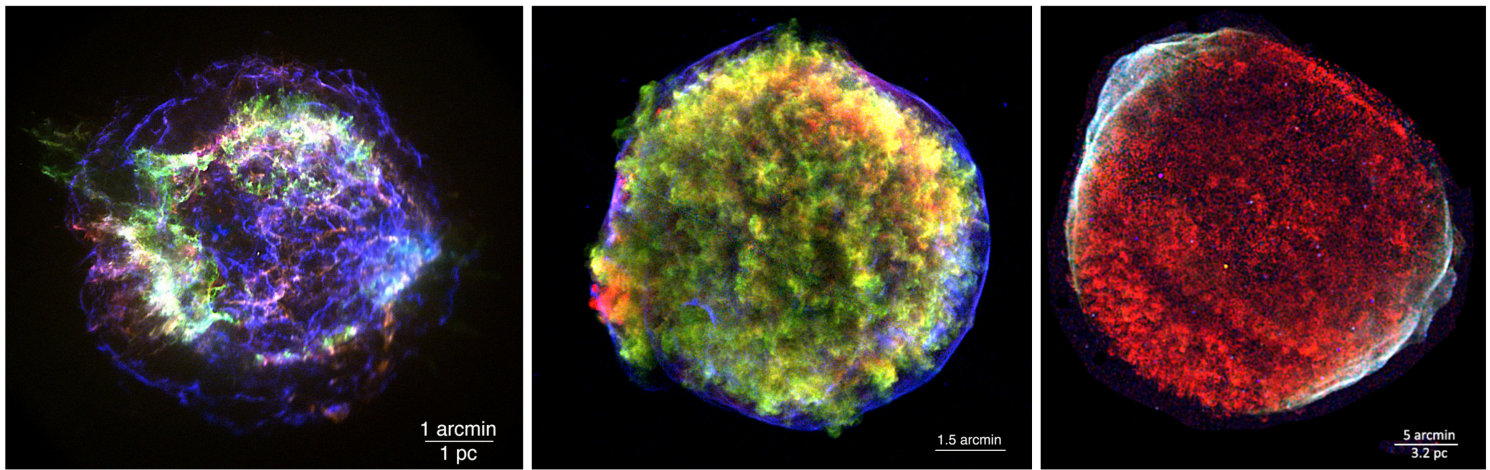

Figure 4. X-ray images of SNRs as observed by Chandra. In all cases the bluish colors represent X-ray synchrotron dominated emission, which in principle should be polarised. (Left) Cassiopeia A as observed in 2004. Red represents Mg XII and Fe L line emission, green Si XIII emission (around $1.85 \mathrm{keV}$ ), and blue is the 4-6 keV continuum band. (Middle) Tycho's SNR, blue is the 4-6 keV band, green is Si XIII (1.75-1.95 keV)and red is Fe L emission (0.8-0.95 keV). (Right) SN 1006, red is the $0.5-0.65 \mathrm{keV}$ band, dominated by O VII oxygen emission, green is $0.85-2.0 \mathrm{keV}$, dominated by line emission from Ne IX, Mg XI and Si XIII (and lower ionisation states), and blue is 3-6.3 keV, dominated by synchrotron emission.

As can be seen in Figure 5 the two different regions, outer filaments and inner filaments, imposed on the input model, were nicely recovered, despite the perpendicular polarisation directions. The weighted scheme recovers within the errors the input polarisation fractions. In Figure 6 shallower and a deeper exposure are shown (top) and simulations with a lower input polarisation fraction at the outer filaments (bottom). The top left image shows that polarisation signals can still be detected with an exposure of $1 \mathrm{Ms}$, but only for a limited set of pixels and at a courser binning level. Apparently the bright western inner part filaments hamper detection of polarisation of the outer shock in this direction. The right image ( $8 \mathrm{Ms}$ ) shows that with enough statistics the measured polarisation fractions are close to the input values, except for the western region again, where the PSF mixes signals from the inner and outer regions. For lower polarisations levels of 5\% the PSF destroys the polarisation signal of the outer filaments. 
Stokes Q

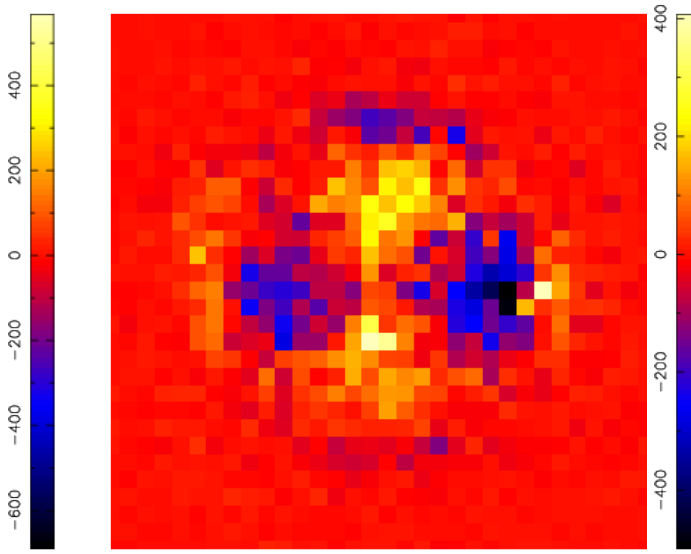

Stokes I

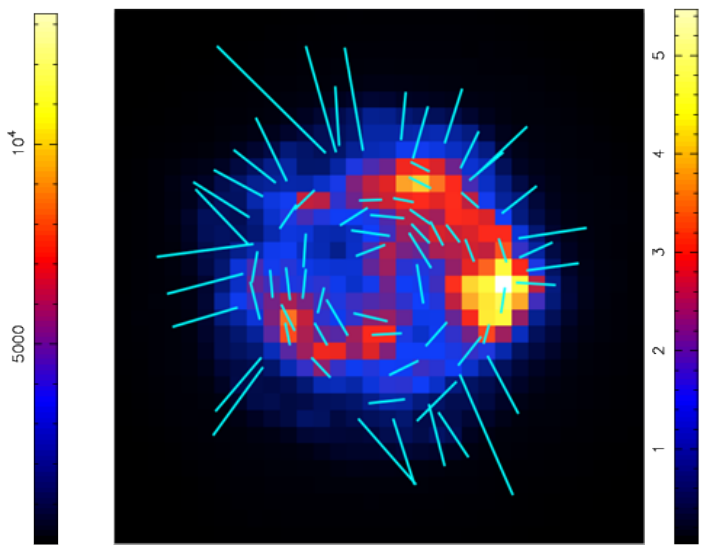

Polarisation Fraction

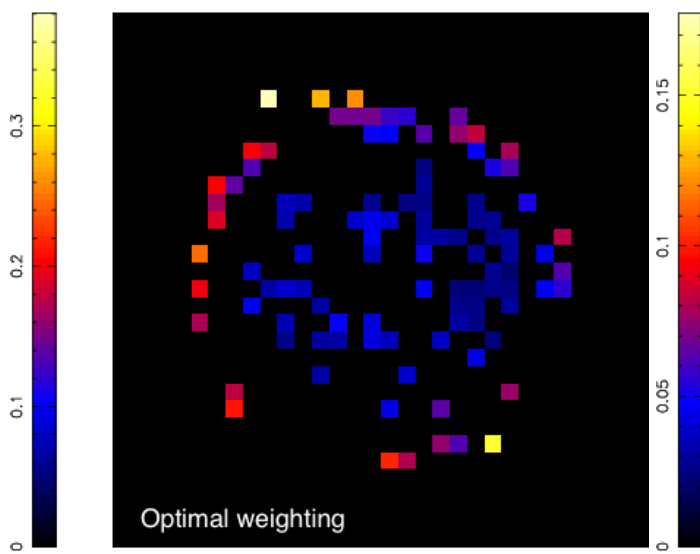

Stokes U

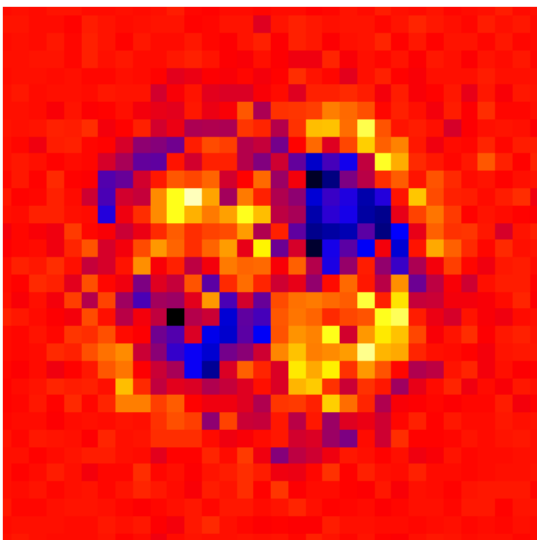

Significance

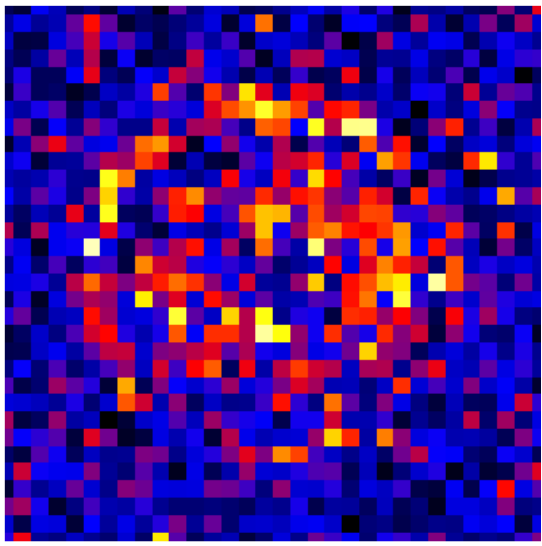

Polarisation Fraction

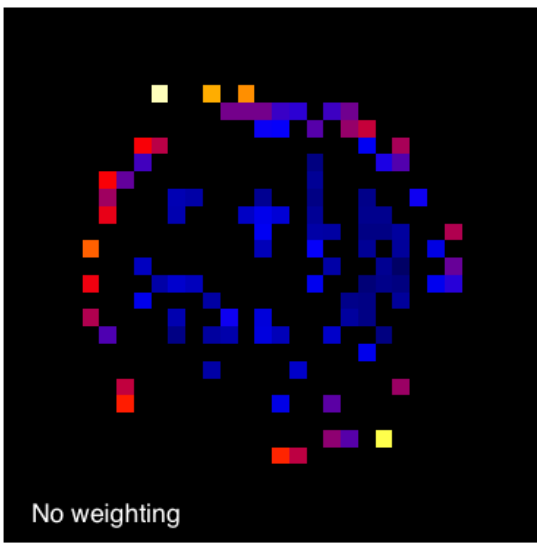

Figure 5. Monte Carlo simulations for Cassiopeia A based on $2 \mathrm{Ms}$ observation with XIPE using photons in the $4-6 \mathrm{keV}$ band. The pixel size is $16^{\prime \prime}$. See the labels for the image identifications. The Stokes I image shows the polarisation E-vector directions, with the length of the line proportional to the polarisation fraction. For the polarisation fractions (lower two images) only pixels for which the polarisation signal to noise ratio is larger than 3 are indicated. Spurious "detections" outside the border of Cas A (see significance map) were ignored. All except the last image (lower right) are based on the weighted scheme for determining the stokes parameters. The last image gives the polarisation fraction without corrections. Visually it is similar to the image in the lower left, but its colour scaling is different. 
Polarisation Fraction

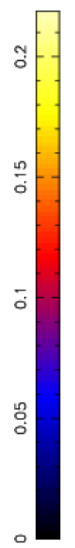

.

Polarisation Fraction
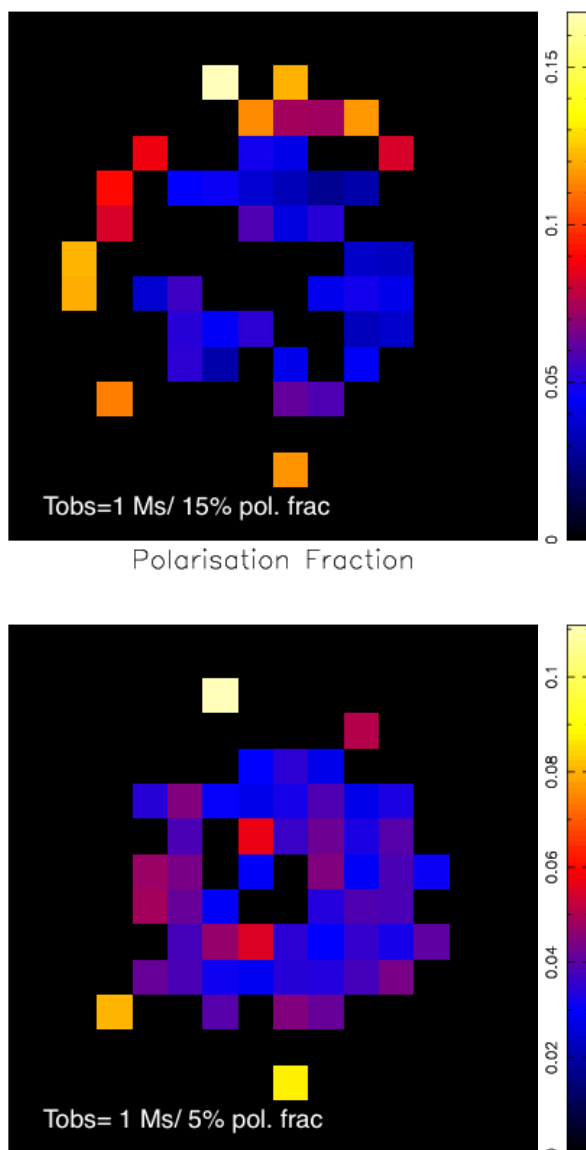

Polarisation Fraction

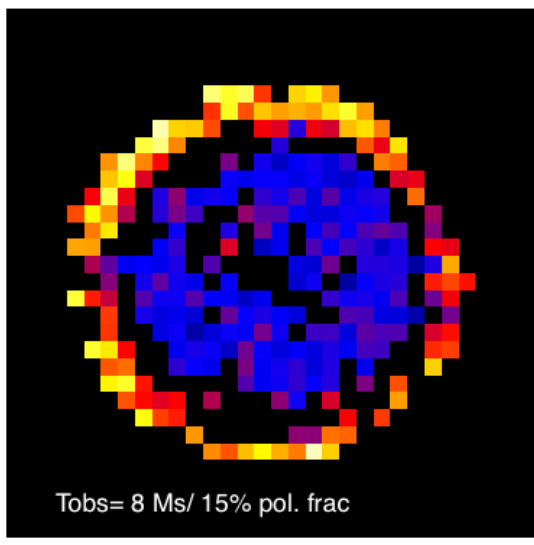

Polarisation Fraction

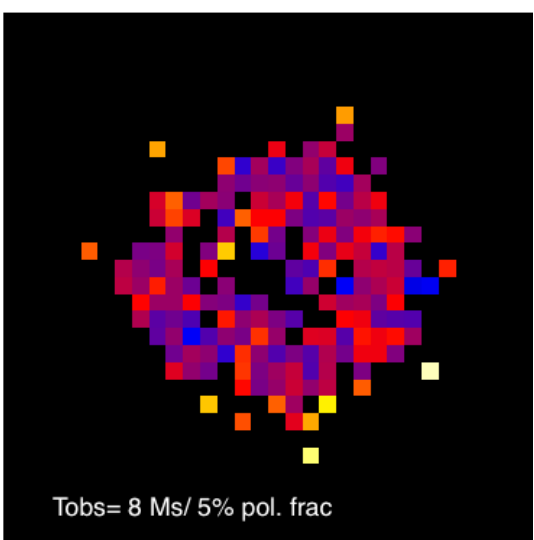

Figure 6. (Top) Polarisation fractions for simulations of Cas A with 1 Ms ((left), pixel size $\left.32^{\prime \prime}\right)$ and $8 \mathrm{Ms}$ (right) exposures. (Bottom) Simulated polarisation fractions, but now the outer filaments have input polarisation fractions of $5 \%$. Both in the $2 \mathrm{Ms}$ (left) and $8 \mathrm{Ms}$ (right) the filaments are not detected as a coherent structure.

\subsection{Tycho's SNR (SN 1572)}

Tycho's SNR has a diameter of $9^{\prime}$, which still fits within the field of view of the XIPE/IXPE and eXTP detectors. Unlike for Cas A, the X-ray synchrotron emission seems to be mostly associated with the forward shock, forming a narrow filament marking the shock front (the blueish/purplish structure Figure 4 centre). Apart from the strikingly thin outer filament, there are a few peculiarly shaped continuum features more toward the centre, which may be outer regions projected to the inside. The best known, and most discussed structure is known as the "Tycho Stripes" on the western side, whose regular structure have been suggested to represent some coherent magnetic field structure shaped by $10^{15} \mathrm{eV}$ cosmic rays [48]. Another feature is an arc-like structure in the southeast.

For this SNR we used the method of input maps (Figure 7 top row) for the polarisation structure. For our simulation of the $4-6 \mathrm{keV}$ band, we used the method of input maps (Figure 7 top row) for the polarisation structure. For the simulated observation time we used 2 Ms. Based on the radio polarisation (Table 1) most of the continuum was assumed to be $7 \%$ polarised, but we assumed that the brightest X-ray continuum features have a polarisation fraction of $25 \%$. This includes a large part of the outer filament, as well as the stripes. The magnetic field was assumed to be radially structured over the whole remnant (the polarisation vectors are then tangential).

As can be seen in Figure 7 for the brightest features the polarisation signal is recovered, but the weaker interior polarisation is not, except for regions associated with the stripes and the arc. Even if we 
increase the exposure time to $8 \mathrm{Ms}$, we cannot detect the interior, and a few high significant pixels are probably caused by scattering from high polarisation structures by the PSF. It should be remembered, however, that the X-ray synchrotron emission from Tycho's SNR is highly limbbrightened and the 4-6 keV continuum surface brightness of the interior is low.

Pol. fraction mode

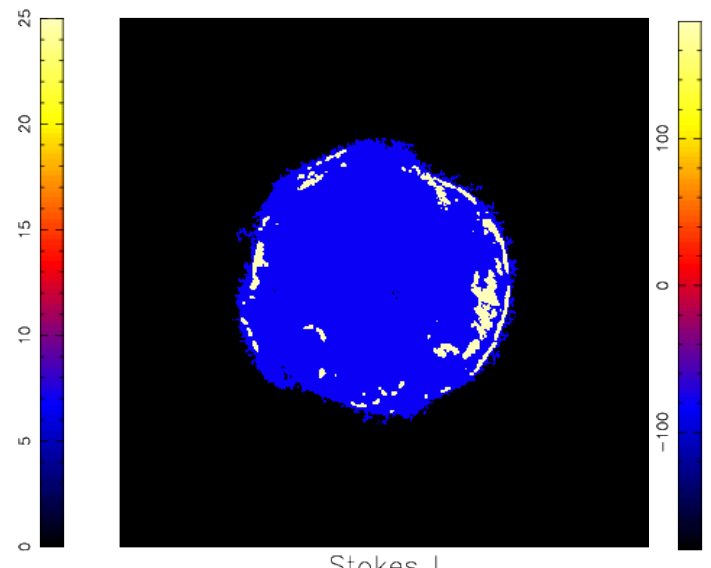

Stokes |
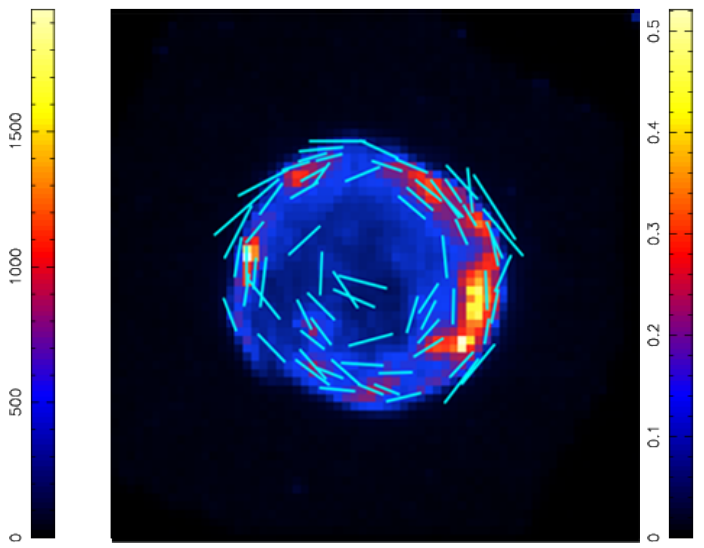

Polarisation angle model

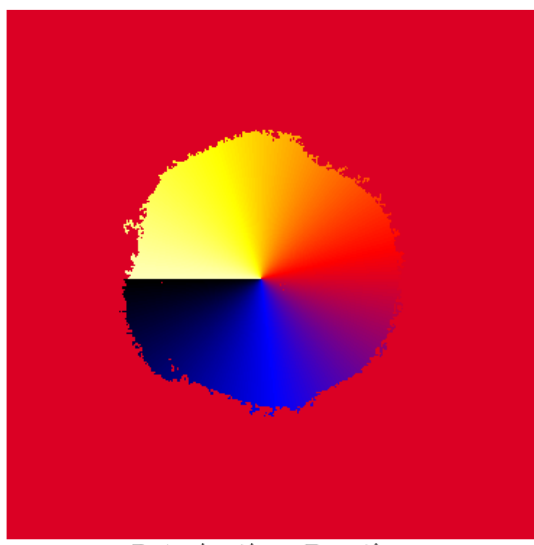

Polarisation Fraction

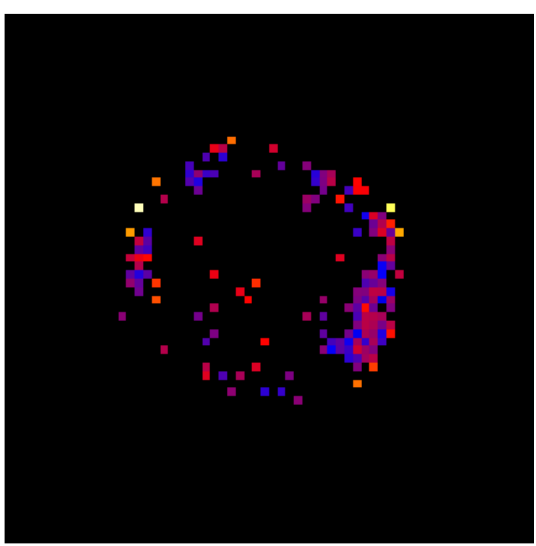

Figure 7. Simulations using a toy input model for Tycho's SNR, based on the Chandra $4-6 \mathrm{keV}$ image and input maps of polarisation fraction and angle, and assuming an observation time of 2 Ms. The output images are binned to $16^{\prime \prime}$ pixels. (Top left) Input model for polarisation fraction (in \%). (Top right) model for the polarisation angle (in degrees). (Bottom left) Simulated 4-6 keV count image (Stokes I). (Bottom right) reconstructured polarisation fraction.

\subsection{SN 1006}

The X-ray synchrotron emission from SN 1006 comes from two X-ray limbs in the northeast and southwest [25], respectively, each of which can be roughly covered with a single XIPE/IXPE pointing. The total diameter of SN 1006 is 30'. Unlike for Cas A and Tycho's SNR, the synchrotron emitting regions emit very little thermal radiation, and most emission above $1 \mathrm{keV}$ is synchrotron emission, whereas from the limbs very little thermal radiation is emitted in the first place. So X-ray polarisation studies do not have to be confined to the $4-6 \mathrm{keV}$ continuum dominated band. For that reason we set-up the Monte Carlo simulations of SN 1006 for the 2-8 keV band, using a power-law spectrum (Table 2) and an assumed polarisation fraction of $17 \%$, based on radio observations (Table 1 ). Since the effective area at $2 \mathrm{keV}$ is larger than at $4 \mathrm{keV}$, and a wider spectral coverage is used, the exposure time for each position was taken to be $1 \mathrm{Ms}$, shorter than for Cas A and Tycho's SNR.

Given that a broader energy coverage can be used, SN 1006 is an ideal target to test how the weighting method (Section 2) affect the measured results. The upper panels of Figure 8 show 
the simulated polarisation fractions of SN 1006 assuming radial magnetic fields, using the three aforementioned weighting methods (left: no weighting; middle: corrected for $\mu$; right: an optimal weighting). Only the data with $\mathrm{S} / \mathrm{N}>4$ are shown in the images and a spurious data point was removed. Here the optimal weighting is found to provide more bins ( 85 pixels) with high polarisation significance ( $\mathrm{S} / \mathrm{N}>3-4)$ than for the corrected method (75 significantly detected pixels).

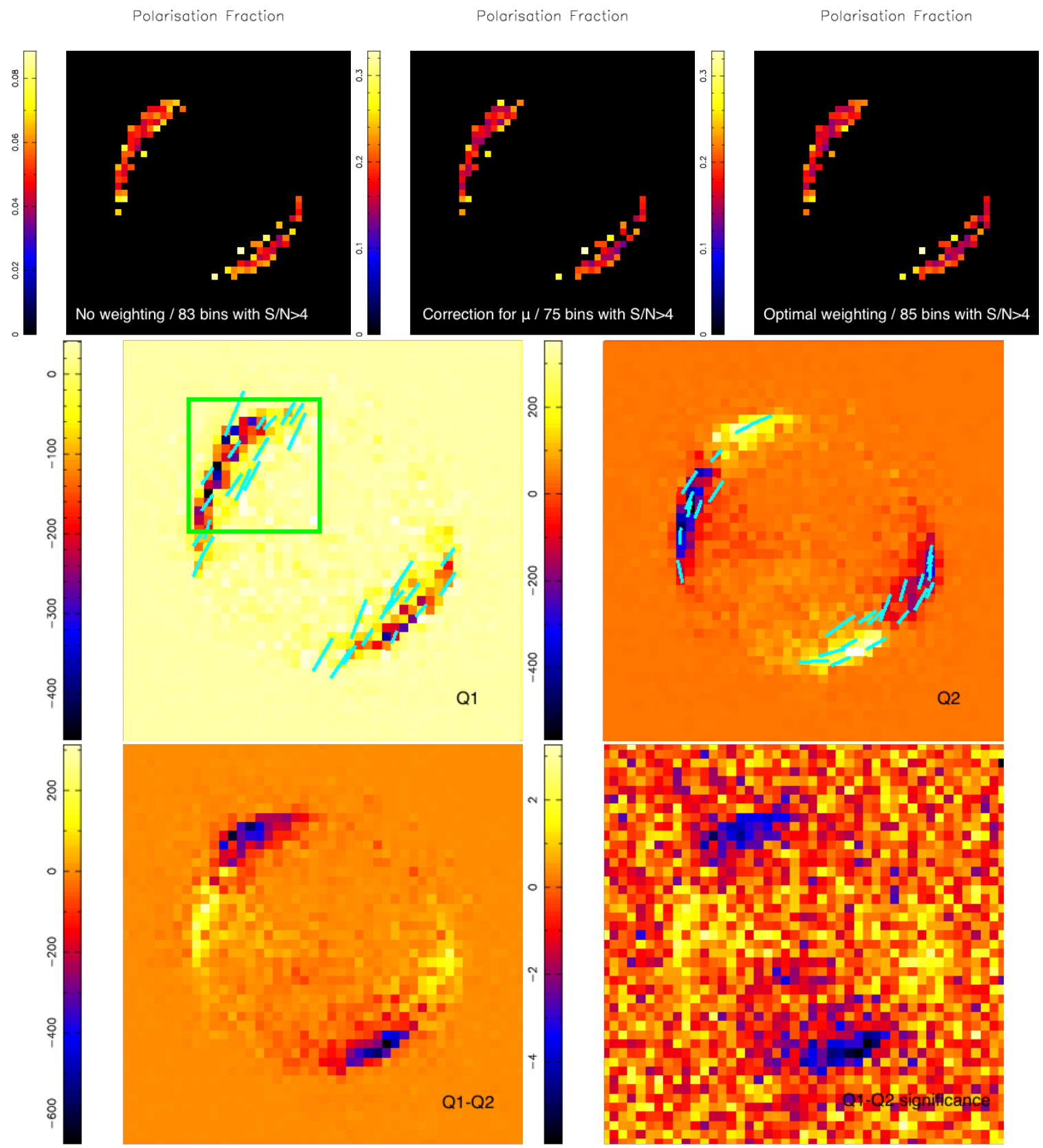

Figure 8. Monte Carlo simulations of SN 1006 in 2-8 keV band based on $1 \mathrm{Ms}$ exposure of each position with XIPE. The output images are binned to $64^{\prime \prime}$ pixels. (Top panels) the polarisation fractions corresponding to the three weighting methods: no weighting, correction for $\mu$ and an optimal weighting. A radial magnetic field is assumed for the two images. (Middle panels) the simulated Stokes $Q$ images using optimal weighting for parallel (left) and radial magnetic field (right) configurations, respectively, overlaid with polarisation vectors (cyan bars) and the field of view of XIPE (green box). (Bottom panels) a difference map for the $Q$ images in the middle panels and the significance map of the difference image on the left. 
The magnetic fields in SN 1006 are likely to be predominantly radial, but the synchrotron morphology has also been explained by invoking parallel magnetic field lines oriented in the northeast-southwest direction [37,49], reflecting the large scale magnetic fields at the location of SN 1006. These two magnetic field configurations (radial and parallel) can be significantly distinguished with a 1 Ms X-ray polarisation measurement, as shown in the middle and bottom panels of Figure 8 . The simulated images of Stokes $Q$ for parallel $\left(Q_{1}\right)$ and radial $\left(Q_{2}\right)$ B-configurations reveal significantly different patterns, as also indicated by the the difference image $Q_{1}-Q_{2}$. The significance image of $Q_{1}-Q_{2}$ is obtained as $\left(Q_{1}-Q_{2}\right) /\left(\sqrt{\operatorname{Var}\left(Q_{1}\right)+\operatorname{Var}\left(Q_{2}\right)}\right)$.

\section{Conclusions}

We have explored here both the science case for X-ray polarisation studies of young SNRs, as well as exploring the practical issue of obtaining X-ray polarisation maps with future instruments on board XIPE, IXPE and eXTP.

In particular, we advocated to use estimators of the Stokes parameters based on summations of the sine and cosine of the photon-electron ejection angles. We expanded on the work of Kislat et al. [14] by also including an energy dependent correction factor, which corrects for instrumental effects, thereby providing estimates of the actual source polarisation fraction. The method is illustrated with simulations of X-ray polarisation maps of young SNRs, but it can also be used for X-ray polarisation timing and spectroscopy studies.

Our simulations show that long exposure times, $1-2 \mathrm{Ms}$, will be needed to detect polarisation signals from young SNRs, but for the important SNRs Cas A, Tycho's SNR and SN 1006 the polarisation signals can be obtained for polarisation fractions comparable to the radio detected polarisation fractions. The long exposure times are necessary to acquire sufficient polarisation statistics, which is more critical than for simple imaging. This is also due to the fact that the GPD detectors have relatively small modulation factors and low quantum efficiencies (less than $30 \%$ above $2 \mathrm{keV}$, [50]). So for X-ray polarisation measurements of astrophysical sources in general, longer exposure times (>100 ks) are to be expected than for imaging spectroscopy observations (typically a few $10 \mathrm{ks}$ ). The number of sources to be observed during the span of the mission is, therefore, necessarily limited. Note that in the yellowbook for XIPE and its mock observation plan these long observation times, including Ms observations of SNRs, were already taken into account.

For SNRs the additional complication is that one needs to rely on the 4-6 keV band to reliably isolate the synchrotron continuum component, since below $4 \mathrm{keV}$ line emission from magnesium, silicon, sulphur, argon and calcium dominate the emission, and above $\sim 6.3 \mathrm{keV}$ iron-K line emission becomes important. However, for isolated synchrotron filaments in certain regions of Cas A and Tycho, one may be able to use the full X-ray band available for polarisation studies (roughly $1.5-8 \mathrm{keV}$ ), or single out isolated spectral regions with weak line contributions. As shown in this publication, for SN 1006 one can already use the $2-8 \mathrm{keV}$ band, as the synchrotron dominated outer rims have only weak line emission.

The X-ray polarisation fractions adopted for our simulations are based on radio polarisation measurement, but the X-ray polarisation fractions may well be different, as the emitting volumes are smaller and the spectral slopes are steeper (both boosting the polarisation fraction), but also comes from regions close to the shock front, where magnetic field turbulence may be higher (which would lead to lower polarisation fractions). The uncertainty on the X-ray polarisation fraction to be expected, as well as the impact the detection of $\mathrm{X}$-ray polarisation would have on our understanding of magnetic field turbulence and particle acceleration efficiency in young SNRs, highlight the need for X-ray polarisation missions.

Acknowledgments: P.Z. acknowledges the support from the NWO Veni Fellowship, grant No. 639.041 .647 and NSFC grants 11503008 and 11590781.

Author Contributions: J.V. has developed the xpolim X-ray polarisation simulation code, and has developed the mathematical equations. The simulations themselves were carried out by J.V. and P.Z. together. 
Conflicts of Interest: The founding sponsors had no role in the design of the study; in the collection, analyses, or interpretation of data; in the writing of the manuscript, and in the decision to publish the results.

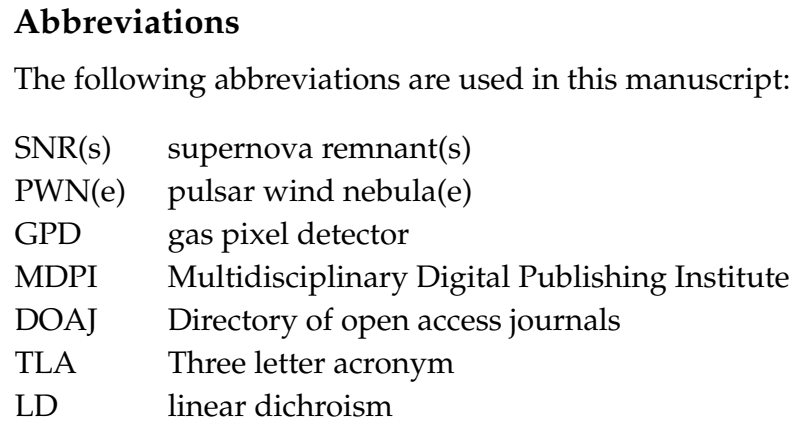

\section{References}

1. Vink, J. Supernova remnants: The X-ray perspective. Astron. Astrophys. Rev. 2012, 20, 49.

2. Novick, R.; Weisskopf, M.C.; Berthelsdorf, R.; Linke, R.; Wolff, R.S. Detection of X-Ray Polarization of the Crab Nebula. Astrophys. J. Lett. 1972, 174, L1-L8.

3. Weisskopf, M.C.; Cohen, G.G.; Kestenbaum, H.L.; Long, K.S.; Novick, R.; Wolff, R.S. Measurement of the X-ray polarization of the Crab Nebula. Astrophys. J. Lett. 1976, 208, L125-L128.

4. Weisskopf, M.C.; Silver, E.H.; Kestenbaum, H.L.; Long, K.S.; Novick, R. A precision measurement of the X-ray polarization of the Crab Nebula without pulsar contamination. Astrophys. J. Lett. 1978, 220, L117-L121.

5. Oort, J.H.; Walraven, T. Polarization and composition of the Crab nebula. Bull. Astron. Neth. 1956, 12, 285.

6. Mayer, C.H.; Hollinger, J.P. Polarized Brightness Distribution Over Cassiopeia a, the Crab Nebula, and Cygnus a at $1.55 \mathrm{~cm}$ Wavelength. Astrophys. J. 1968, 151, 53.

7. Bietenholz, M.F.; Kronberg, P.P. The magnetic field of the Crab Nebula and the nature of its 'jet'. Astrophys. J. Lett. 1990, 357, L13-L16.

8. Moran, P.; Kyne, G.; Gouiffès, C.; Laurent, P.; Hallinan, G.; Redfern, R.M.; Shearer, A. A recent change in the optical and $\gamma$-ray polarization of the Crab nebula and pulsar. Mon. Not. R. Astron. Soc. 2016, 456, 2974-2981.

9. Costa, E.; Soffitta, P.; Bellazzini, R.; Brez, A.; Lumb, N.; Spandre, G. An efficient photoelectric X-ray polarimeter for the study of black holes and neutron stars. Nature 2001, 411, 662-665.

10. Bellazzini, R.; Spandre, G.; Minuti, M.; Baldini, L.; Brez, A.; Latronico, L.; Omodei, N.; Razzano, M.; Massai, M.M.; Pesce-Rollins, M.; et al. A sealed Gas Pixel Detector for X-ray astronomy. Nucl. Instrum. Methods Phys. Res. A 2007, 579, 853-858.

11. Soffitta, P.; Barcons, X.; Bellazzini, R.; Braga, J.; Costa, E.; Fraser, G.W.; Gburek, S.; Huovelin, J.; Matt, G.; Pearce, M.; et al. XIPE: The X-ray imaging polarimetry explorer. Exp. Astron. 2013, 36, 523-567.

12. Weisskopf, M.C.; Ramsey, B.; O’Dell, S.; Tennant, A.; Elsner, R.; Soffitta, P.; Bellazzini, R.; Costa, E.; Kolodziejczak, J.; Kaspi, V.; et al. The Imaging X-ray Polarimetry Explorer (IXPE). In Space Telescopes and Instrumentation 2016: Ultraviolet to Gamma Ray; SPIE: Bellingham, WA, USA, 2016; Volume 9905, p. 990517.

13. Zhang, S.N.; Feroci, M.; Santangelo, A.; Dong, Y.W.; Feng, H.; Lu, F.J.; Nandra, K.; Wang, Z.S.; Zhang, S.; Bozzo, E.; et al. eXTP: Enhanced X-ray Timing and Polarization mission. In Space Telescopes and Instrumentation 2016: Ultraviolet to Gamma Ray; SPIE: Bellingham, WA, USA, 2016; Volume 9905, p. 99051Q.

14. Kislat, F.; Clark, B.; Beilicke, M.; Krawczynski, H. Analyzing the data from X-ray polarimeters with Stokes parameters. Astropart. Phys. 2015, 68, 45-51.

15. Muleri, F.; Soffitta, P.; Baldini, L.; Bellazzini, R.; Brez, A.; Costa, E.; Fabiani, S.; Krummenacher, F.; Latronico, L.; Lazzarotto, F.; et al. Spectral and polarimetric characterization of the Gas Pixel Detector filled with dimethyl ether. Nucl. Instrum. Methods Phys. Res. A 2010, 620, 285-293.

16. De Ona Wilhelmi, E.; Vink, J.; Bykov, A.; Zanin, R.; Bucciantini, N.; Amato, E.; Bandiera, R.; Olmi, B.; Uvarov, Y.; XIPE Science Working Group. Unveiling the magnetic structure of VHE SNRs/PWNe with XIPE, the X-ray imaging-polarimetry explorer. In Proceedings of the 6th International Symposium on High Energy Gamma-Ray Astronomy, Heidelberg, Germany, 11-15 July 2017; Volume 1792, p. 070023.

17. Meier, P. Variance of a weighted mean. Biometrics 1953, 9, 59-73.

18. Shahar, D. Minimizing the Variance of a Weighted Average. Open J. Stat. 2017, 7, 216-224. 
19. Fabiani, S.; Costa, E.; Del Monte, E.; Muleri, F.; Soffitta, P.; Rubini, A.; Bellazzini, R.; Brez, A.; de Ruvo, L.; Minuti, M.; et al. The Imaging Properties of the Gas Pixel Detector as a Focal Plane Polarimeter. Astrophys. J. Suppl. Ser. 2014, 212, 25.

20. Morrison, R.; McCammon, D. Interstellar photoelectric absorption cross sections, $0.03-10 \mathrm{keV}$. Astrophys. J. 1983, 270, 119-122.

21. Shklovsky, J.S. Supernovae as a Source of Cosmic Rays. In Proceedings of the Liege International Astrophysical Colloquia, Liege, Belgium, 10-12 September 1953; Volume 5, p. 515.

22. Malkov, M.A.; Drury, L. Nonlinear theory of diffusive acceleration of particles by shock waves. Rep. Prog. Phys. 2001, 64, 429-481.

23. Bell, A.R. Turbulent amplification of magnetic field and diffusive shock acceleration of cosmic rays. Mon. Not. R. Astron. Soc. 2004, 353, 550-558.

24. Marcowith, A.; Bret, A.; Bykov, A.; Dieckman, M.E.; O’C Drury, L.; Lembège, B.; Lemoine, M.; Morlino, G.; Murphy, G.; Pelletier, G.; et al. The microphysics of collisionless shock waves. Rep. Prog. Phys. 2016, 79, 046901.

25. Koyama, K.; Petre, R.; Gotthelf, E.V.; Hwang, U.; Matsuura, M.; Ozaki, M.; Holt, S.S. Evidence for Shock Acceleration of High-Energy Electrons in the Supernova Remnant SN 1006. Nature 1995, 378, 255-258.

26. Aharonian, F.A.; Atoyan, A.M. On the origin of TeV radiation of SN 1006. Astron. Astrophys. 1999, 351, 330-340.

27. Vink, J.; Laming, J.M. On the magnetic fields and particle acceleration in Cassiopeia A. Astrophys. J. 2003, $584,758-769$.

28. Helder, E.A.; Vink, J.; Bykov, A.M.; Ohira, Y.; Raymond, J.C.; Terrier, R. Observational Signatures of Particle Acceleration in Supernova Remnants. Space Sci. Rev. 2012, 173, 369-431.

29. Dickel, J.R.; Jones, E.M. Magnetic Fields in Young Supernova Remnants. In Galactic and Intergalactic Magnetic Fields; Beck, R., Wielebinski, R., Kronberg, P.P., Eds.; IAU Symposium: Paris, France, 1990; Volume 140, p. 81.

30. Dickel, J.R.; Milne, D.K. Magnetic fields in supernova remnants. Aust. J. Phys. 1976, 29, 435-460.

31. Zirakashvili, V.N.; Ptuskin, V.S. Diffusive Shock Acceleration with Magnetic Amplification by Nonresonant Streaming Instability in Supernova Remnants. Astrophys. J. 2008, 678, 939-949.

32. West, J.L.; Jaffe, T.; Ferrand, G.; Safi-Harb, S.; Gaensler, B.M. When Disorder Looks Like Order: A New Model to Explain Radial Magnetic Fields in Young Supernova Remnants. Astrophys. J. Lett. 2017, 849, L22.

33. Milne, D.K.; Dickel, J.R. 5 GHz polarization observations of 33 galactic radio sources. Aust. J. Phys. 1975, 28, 209-230.

34. Ginzburg, V.L.; Syrovatskii, S.I. Cosmic Magnetobremsstrahlung (synchrotron Radiation). Annu. Rev. Astron. Astrophys. 1965, 3, 297-350.

35. Bykov, A.; Uvarov, Y. Polarized synchrotron X-ray emission from supernova shells: XIPE/IXPE perspective. In Journal of Physics Conference Series; IOP Publishing: Bristol, UK, 2017; Volume 932, p. 012051.

36. Dickel, J.R.; Strom, R.G.; Milne, D.K. The Radio Structure of the Supernova Remnant G315.4-2.3 (MSH 14-63). Astrophys. J. 2001, 546, 447-454.

37. Reynoso, E.M.; Hughes, J.P.; Moffett, D.A. On the Radio Polarization Signature of Efficient and Inefficient Particle Acceleration in Supernova Remnant SN 1006. Astron. J. 2013, 145, 104.

38. Dickel, J.R.; van Breugel, W.J.M.; Strom, R.G. Radio structure of the remnant of Tycho's supernova (SN 1572). Astron. J. 1991, 101, 2151-2159.

39. DeLaney, T.; Koralesky, B.; Rudnick, L.; Dickel, J.R. Radio Spectral Index Variations and Physical Conditions in Kepler's Supernova Remnant. Astrophys. J. 2002, 580, 914-927.

40. Braun, R.; Gull, S.F.; Perley, R.A. Physical process which shapes Cassiopeia A. Nature 1987, 327, $395-398$.

41. Anderson, M.C.; Keohane, J.W.; Rudnick, L. The polarization and depolarization of radio emission from supernova remnant Cassiopeia A. Astrophys. J. 1995, 441, 300-306.

42. De Horta, A.Y.; Filipovic, M.D.; Crawford, E.J.; Stootman, F.H.; Pannuti, T.G.; Bozzetto, L.M.; Collier, J.D.; Sommer, E.R.; Kosakowski, A.R. Radio-Continuum Emission from the Young Galactic Supernova Remnant G1.9 + 0.3. Serbian Astron. J. 2014, 189, 41-51.

43. Bamba, A.; Fukazawa, Y.; Hiraga, J.S.; Hughes, J.P.; Katagiri, H.; Kokubun, M. Suzaku Wide-Band Observations of SN 1006. Publ. Astron. Soc. Jpn. 2008, 60, 153-161.

44. Helder, E.A.; Vink, J. Characterizing the Nonthermal Emission of Cassiopeia A. Astrophys. J. 2008, 686, 1094-1102. 
45. Uchiyama, Y.; Aharonian, F.A. Fast Variability of Nonthermal X-Ray Emission in Cassiopeia A: Probing Electron Acceleration in Reverse-Shocked Ejecta. Astrophys. J. Lett. 2008, 677, L105-L108.

46. Gotthelf, E.V.; Koralesky, B.; Rudnick, L.; Jones, T.W.; Hwang, U.; Petre, R. Chandra detection of the forward and reverse shocks in Cassiopeia A. Astrophys. J. Lett. 2001, 552, L39-L43.

47. Hwang, U.; Laming, J.M.; Badenes, C.; Berendse, F.; Blondin, J.; Cioffi, D. A million second Chandra view of Cassiopeia A. The Astrophysical Journal Letters, 615(2), L117. A Million Second Chandra View of Cassiopeia A. Astrophys. J. Lett. 2004, 615, L117-L120.

48. Eriksen, K.A.; Hughes, J.P.; Badenes, C.; Fesen, R.; Ghavamian, P.; Moffett, D.; Plucinksy, P.P.; Rakowski, C.E.; Reynoso, E.M.; Slane, P. Evidence for Particle Acceleration to the Knee of the Cosmic Ray Spectrum in Tycho's Supernova Remnant. Astrophys. J. Lett. 2011, 728, L28.

49. Rothenflug, R.; Ballet, J.; Dubner, G.; Giacani, E.; Decourchelle, A.; Ferrando, P. Geometry of the non-thermal emission in SN 1006. Astron. Astrophys. 2004, 425, 121-131.

50. Bellazzini, R.; Angelini, F.; Baldini, L.; Bitti, F.; Brez, A.; Cavalca, F.; Del Prete, M.; Kuss, M.; Latronico, L.; Omodei, N.; et al. Gas pixel detectors for X-ray polarimetry applications. Nucl. Instrum. Methods Phys. Res. A 2006, 560, 425-434.

(C) 2018 by the authors. Licensee MDPI, Basel, Switzerland. This article is an open access article distributed under the terms and conditions of the Creative Commons Attribution (CC BY) license (http:/ / creativecommons.org/licenses/by/4.0/). 